\title{
Investigation of Modulators in OTDM system
}

\author{
Jameel Ahamed \\ Department of Electronics Engg \\ ZHCET AMU, Aligarh
}

\author{
Amir Khan \\ Department of Electronics Engg \\ ZHCET AMU, Aligarh
}

\author{
Mumtaz Ahmed \\ Department of computer Engg \\ Jamia Millia Islamia, New Delhi
}

\begin{abstract}
With the ever-increasing demand for higher speeds and larger capacity brought about by rapid data growth on the Internet, interest in optical time division multiplexing has been growing rapidly in recent years due to its high speed and ability to overcome the "electronic bottleneck" offered by today's electronic components. Optical time division multiplexing (OTDM) transmits multiple data channels in the form of ultra-short duration optical pulses which are interleaved into a single high-speed data stream by accurate control of their relative delay in the time domain. OTDM transmission system has been a topic of continuous research due to their unique advantages over conventional Pulse sources based transmissions. Among various key high repetition rate, limited pulse source, pulse range still remains as the issues that require further optimization. The performance of OTDM system is analyzed by using BER analyzer in which Q factor is to be measured. In OTDM system the maximum $\mathrm{Q}$ factor can be achieved by using different methods which are increasing laser power, using various.. We will focus on performance metrics and parameters used in the topology. We also focused on various modulators related to the topology.
\end{abstract}

\section{Keywords}

OTDM, BER

\section{INTRODUCTION}

Although an optical fibre is a very wideband medium for information transmission, practically it is impossible to modulate the full optical bandwidth at once. This is because an optical signal is initially generated from an electrical data pattern and converted back into an electrical signal after transmission for the recovery of the transmitted data [1]. With the ever-increasing demand for higher speeds and larger capacity brought about by rapid data growth on the Internet, interest in optical time division multiplexing has been growing rapidly in recent years due to its high speed and ability to overcome the "electronic bottleneck" offered by today's electronic components. In comparison to Wavelength Division Multiplexing (WDM) where multiplexing occurs in the frequency domain, Optical Time-Division Multiplexing (OTDM) transmits multiple data channels in the form of ultrashort duration optical pulses which are interleaved into a single high-speed data stream by accurate control of their relative delay in the time domain[2]. By using OTDM technique very high capacity of data over optical fiber can be achieved. These type of systems can provides higher speed than electronic components, several technologies are needed to realize in order to achieve higher speed in OTDM systems. These include high repetition rate ultra-short pulse sources, high speed demultiplexing, clock recovery and accurate dispersion compensation. Many of these technologies are still at the research stage, which makes high-speed OTDM currently a relatively expensive transmission solution.
Regardless of this, OTDM is still an alternative way to increase the transmission capacity of a fiber system. Compared to conventional WDM transmission systems, it may offer several advantages [2]. In terms of transmission performance, since only one wavelength is used in a pure OTDM system, the gain tilt problem and dispersion tilt problem $[3,4]$ associated with wide-band WDM transmission can be eliminated. Also, the major limiting nonlinear effects for WDM systems, such as four wave mixing (FWM) and Stimulated Raman scattering (SRS) can be avoided [5].

The basic principle of this technology is to multiplex a number of low bit rate optical channels in time domain as illustrated in Figure 1. At the transmitter side the OTDM all the signals are modulated and then combined by using power combiner in order to travel on the channel. At the receiver, the OTDM signal is separated into original multiple base-rate channels by using power splitter for the BER measurements. OTDM transmission systems require an all-channel independent modulation multiplexer (MUX) and an allchannel simultaneous demultiplexer (DEMUX).

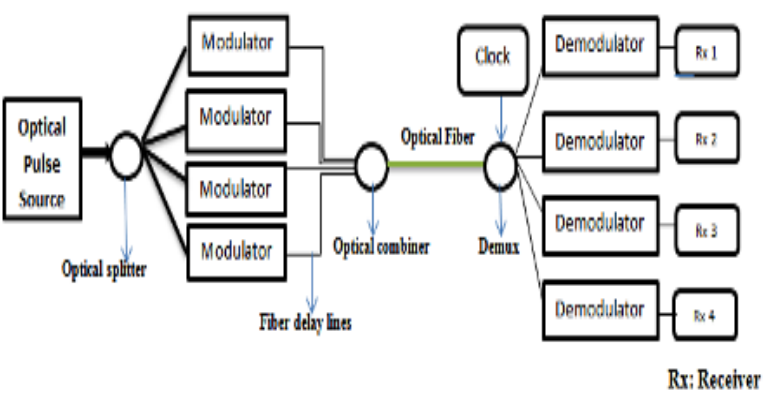

Fig.1: OTDM System

\section{MODULATION}

The huge quantity of data which is utilizing and transmitting daily for using in internet or when using in telephony is created and processed in the electrical domain. On the other hand, we can take the benefit of using light for processing and transporting this data. By using optical communication one can get long haul and high capacity submarine links (from about 1000 to a couple of 10000's km) [6]. Accordingly, the data originating from a large number of users is multiplexed in time in the electrical domain before being transmitted at a high bit rate over optical fibers.

An important aspect of an optical system is therefore the modulation operation, which is used for converting the high bit-rate electrical data signal into the optical domain. Ideal modulation is therefore equivalent to performing a frequency translation from the baseband to an optical carrier frequency, of the order of $193 \mathrm{THz}$ (i.e., $193 \times 1012 \mathrm{~Hz}$ ) for the usual 
$1550 \mathrm{~nm}$ transmission window [6]. In this paper I have used the three types of external modulators which are described below.

\subsection{Amplitude Modulator}

Amplitude modulator is defined as modulation of the signal by varying its amplitude. In this modulator, the optical carrier is modulated externally by the electrical modulation signal. Assuming that the optical input signal is $\mathrm{E}_{\text {in }}$, the following equations describe the behaviour of the model.

$$
E_{\text {out }}(t)=E_{\text {in }}(t) \cdot \sqrt{(\operatorname{Mod}(t))}
$$

Where $E_{\text {out }}(\mathrm{t})$ is the output optical signal and $\operatorname{Mod}(\mathrm{t})$ is defined as

$$
\operatorname{Mod}(t)=(1-M I)+M I \cdot \text { Modulation }(t)
$$

Where MI is the modulation index and modulation $(t)$ is the electrical input signal. The electrical input signal is normalized between 0 and 1[7].

\subsection{Mach-Zehnder Modulator}

The Mach-Zehnder modulator is an intensity modulator based on an interferometric principle. It consists of two $3 \mathrm{~dB}$ couplers which are connected by two waveguides of equal length. By means of an electro-optic effect, an externally applied voltage can be used to vary the refractive indices in the waveguide branches. The different paths can lead to constructive and destructive interference at the output, depending on the applied voltage. Then the output intensity can be modulated according to the voltage[7].

In order to illustrate the principle, we consider the simple interferometric structure represented in Fig.2.1. It is based on a Mach-Zehnder interferometer including one electro-optic material in one of the arms.

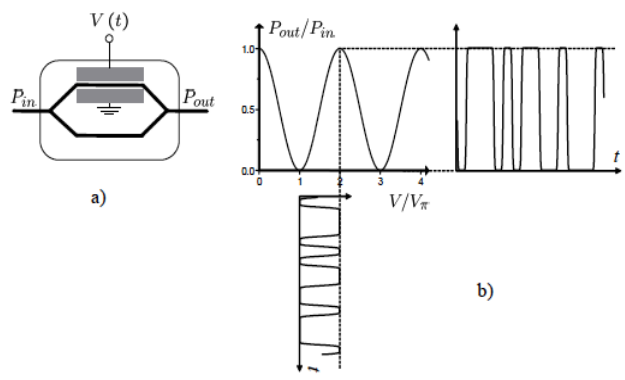

Fig.2: Principle operation of a Mach- Zehnder Modulator.

\subsection{Electro-absorption modulator.}

This type of modulator relies on the fact that the effective bandgap $\mathrm{E}_{\mathrm{g}}$ of a semiconductor material decreases when an external voltage is applied. Consequently, if the frequency of an incoming lightwave is chosen so that its energy $E=h v$ is smaller than the bandgap when no voltage is applied, the material will be transparent. On the other hand, when an external voltage is applied, the effective bandgap will be reduced, meaning that the lightwave will be absorbed by the material when $\mathrm{E}>\mathrm{E}_{\mathrm{g}}[7]$.

\section{PROBLEM DEFINITION}

In OTDM system, at the transmitter side Pseudo random bit sequence generator is used. (PRBS) generates the bits according to order mode. Here PRBS generator with order $\mathrm{k}$ is used to generate a sequence with period of $2^{\wedge} \mathrm{k}-1$. The random sequence then fed to RZ pulse generator to produce electrical $\mathrm{RZ}$ pulse, generates a return to zero coded signal. Rectangle shape is used to determine the shape of edges of the pulse. According to the parameter Rectangle shape, this model can produce pulses with different edge shapes as Exponential, Gaussian, Linear and sine range which can be used as default value.

In OTDM system, modulator is used both at transmitter side and receiver side. At transmitter side it is used to convert electrical signal to optical signal and at receiver side used as demodulator in order to convert optical signal back to electrical signal for BER measurements.

So, our aim is to investigate the best range of the shape of RZ pulse generator in OTDM system when the different modulators are used. The modulators used in my system are Amplitude modulator, Mach-Zehnder modulator and Electroabsorption modulator. All the three modulators are discussed above.

\section{SIMULATION RESULTS AND DISCUSSION}

Multiple simulations for AM, MZM and EAM for OTDM system at an optical wavelength of $1550 \mathrm{~nm}$ are carried out. The results were analysed using BER analyser in which Q factor is to be evaluated.

The system performance is monitored on the basis of BER analyzer and graphs generated from the BER Analyzer for the following system setups.

The designed OTDM system is used for analysis of different modulators and different pulse shapes of the RZ pulse generator. The main motivation behind this is to find the optimum modulator for which $\mathrm{Q}$ factor is greater than or equal to 6 as required by the OFC standard. The system is configured with 4 channels. Using the system simulation setup in the Optisystem9.0 simulator, the value of Q factor is measured by using BER analyzer at the receiver after using different modulators.

Required $Q>=6$ (as per OFC standard).

At Fiber attenuation $=0 \mathrm{ps} / \mathrm{nm} / \mathrm{km}$.

At Laser frequency $=193.1 \mathrm{THz}$.

At Bit rate $=10 \mathrm{~GB} / \mathrm{s}$.

In this paper I have taken into consideration three parts for evaluation.

The different RZ pulse generators used in this setup are Exponential, Gaussian, Linear and Sine. 


\subsection{Amplitude Modulation with different ranges of $R Z$ pulse generator}

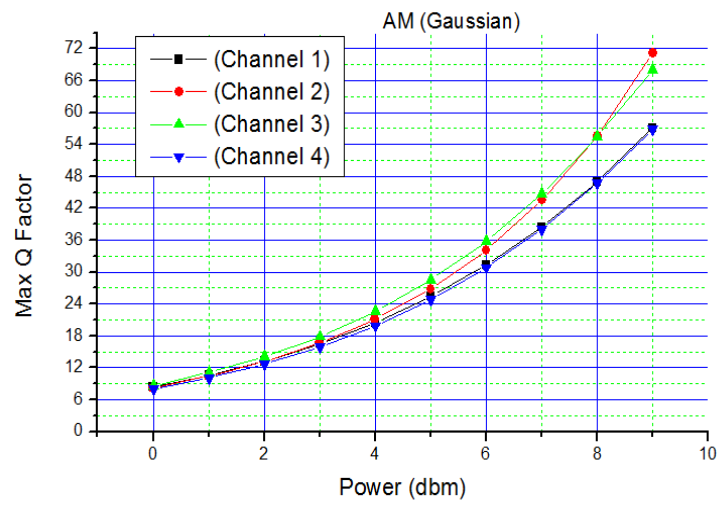

Fig. 3: Max Q Factor verses Power at Gaussian pulse shape of AM.

\subsection{Mach- Zehnder Modulation with} different ranges of $\mathrm{RZ}$ pulse generator

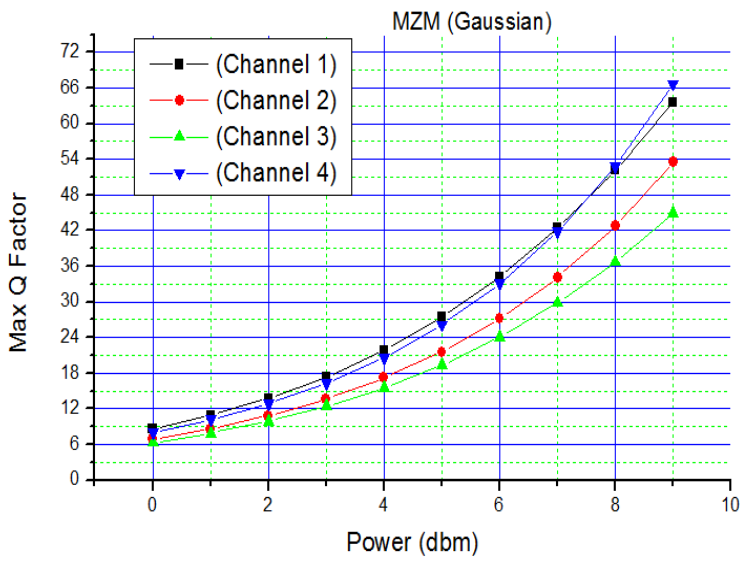

Fig. 4: Max Q Factor verses Power at Gaussian pulse shape of MZM.

\subsection{Electro-absorption Modulation with} different ranges of $\mathrm{RZ}$ pulse generator

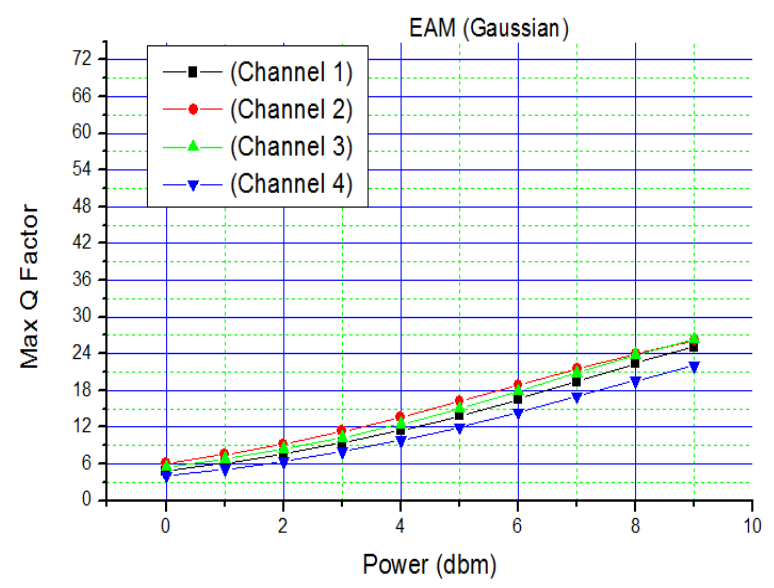

\begin{tabular}{|l|l|r|r|r|}
\hline \multicolumn{1}{|l|}{ Table 1: Q.FACTOR VERSUS POWER for Amplitude } \\
Modulator
\end{tabular}

\begin{tabular}{|c|c|c|c|c|}
\hline $\begin{array}{l}\text { Power } \\
\text { in } \mathrm{dbm}\end{array}$ & Channel 1 & Channel 2 & $\begin{array}{l}\text { Channel } \\
3\end{array}$ & $\begin{array}{l}\text { Channel } \\
4\end{array}$ \\
\hline 0 & 8.60403 & 6.81934 & 6.29243 & 8.0453 \\
\hline 1 & 10.8932 & 8.58896 & 7.89995 & 10.1819 \\
\hline 2 & 13.7657 & 10.8183 & 9.90837 & 12.8849 \\
\hline 3 & 17.3568 & 13.6251 & 12.4079 & 16.3053 \\
\hline 4 & 21.8303 & 17.1556 & 15.5099 & 20.6338 \\
\hline 5 & 27.3677 & 21.5885 & 19.3464 & 26.1167 \\
\hline 6 & 34.155 & 27.1386 & 24.057 & 33.0525 \\
\hline 7 & 42.3718 & 34.0748 & 29.8029 & 41.811 \\
\hline 8 & 52.1677 & 42.7112 & 36.7186 & 52.8368 \\
\hline 9 & 63.6229 & 53.4416 & 44.9183 & 66.639 \\
\hline
\end{tabular}

Fig.5: Max Q Factor verses Power at Gaussian pulse shape of EAM. 


\begin{tabular}{|l|l|l|l|l|}
\hline \multicolumn{5}{|c|}{$\begin{array}{l}\text { Table 3: Q.FACTOR VERSUS POWER FOR Electro- } \\
\text { absorption Modulator }\end{array}$} \\
\hline $\begin{array}{l}\text { Power } \\
\text { in dbm }\end{array}$ & $\begin{array}{l}\text { Channel } \\
1\end{array}$ & $\begin{array}{l}\text { Channel } \\
2\end{array}$ & $\begin{array}{l}\text { Channel } \\
3\end{array}$ & Channel 4 \\
\hline 0 & 4.86434 & 6.03415 & 5.47966 & 4.06213 \\
\hline 1 & 6.08601 & 7.48788 & 6.77677 & 5.10376 \\
\hline 2 & 7.56763 & 9.23256 & 8.35106 & 6.38478 \\
\hline 3 & 9.33771 & 11.2847 & 10.2351 & 7.94294 \\
\hline 4 & 11.424 & 13.6282 & 12.4509 & 9.80257 \\
\hline 5 & 13.8333 & 16.2022 & 15.0073 & 11.9691 \\
\hline 6 & 16.5363 & 18.8925 & 17.852 & 14.3954 \\
\hline 7 & 19.446 & 21.5432 & 20.8516 & 16.9798 \\
\hline 8 & 22.3941 & 23.9907 & 23.7865 & 19.5624 \\
\hline 9 & 25.1499 & 26.1082 & 27.3748 & 21.96 \\
\hline & & & & \\
\hline
\end{tabular}

\section{CONCLUSION}

After analyzing the various evaluated results (Table 1, Table 2, Table 3) and various graphs (Fig.3, Fig.4, Fig.5) we have concluded that keeping the $\mathrm{cw}$ laser frequency and bit rate fixed the best performance is achieved for amplitude modulator for Gaussian pulse range of RZ pulse generator.

1. With the increase in power the maximum $Q$ factor increases. For laser power equal to $0 \mathrm{dbm}$ the maximum $\mathrm{Q}$ factor is above 6 which is just acceptable in OFC standard.
2. By increasing power upto $9 \mathrm{dbm}$ the maximum $\mathrm{Q}$ factor increases.

3. For AM, MZM and EAM the best performing pulse shape is Gaussian pulse shape in which the maximum $Q$ factor is acceptable as per OFC standard.

\section{REFERENCES}

[1] M Arumugam, "Optical Fiber communication- An overview", Pramana- Journal of Physics, Indian Academy of Sciences, Vol. 57, Nos. 5 \& 6. 2001, pp. 849-869.

[2] Fengqiu Wang "Picosecond pulse generation and its applications in high speed OTDM systems".

[3] V. J. Mazurczuyk, et al., "Accumulation of gain tilt in WDM amplified systems due to Raman crosstalk," Photon. Tech. Lett., IEEE, vol. 12, no. 11, pp. 1573 1575, Nov. 2000

[4] D. F. Grosz, et al., "All-Raman ultralong-haul singlewideband DWDM transmission systems with OADM capability," J. Lightwave Technol., vol. 22, no. 2, pp. 423-432, Feb. 2004.

[5] S. Norimatsu, et al., "Waveform distortion due to stimulated Raman scattering in wide-band WDM transmission systems," J. Lightwave Technol., vol. 19, no. 2, pp. 159-171, Feb 2001.

[6] Chirstophe peucheret "Direct and External modulation of light", 2009.

[7] Optiwave, "optisystem9.0 Component Library" , 2010.

[8] John M Senior, "Optical Fiber Communication", Second edition, Prentice Hall of India, 2004.

[9] Sergejs Makovejs High-speed optical fibre transmission using advanced modulation formats.

[10] Jameel Ahamed, Gausia Qazi, Mumtaz Ahmed "Investigation of Sensitivity of Performance to Pulse Shapes in OTDM System", ACEEE, 2013. 